\title{
Automatic segmentation of right ventricular ultrasound images using sparse matrix transform and a level set
}

\author{
Xulei Qin ${ }^{1}$, Zhibin Cong1, and Baowei Fei ${ }^{1,2,3,4}$ \\ ${ }^{1}$ Department of Radiology and Imaging Sciences, Emory University, Atlanta, GA 30329, USA \\ ${ }^{2}$ Department of Mathematics and Computer Science, Emory University, Atlanta, GA 30329, USA \\ ${ }^{3}$ The Joint Department of Biomedical Engineering, Emory University and Georgia Institute of \\ Technology, Atlanta, GA 30329, USA
}

\begin{abstract}
An automatic segmentation framework is proposed to segment the right ventricle (RV) in echocardiographic images. The method can automatically segment both epicardial and endocardial boundaries from a continuous echocardiography series by combining sparse matrix transform, a training model, and a localized region-based level set. First, the sparse matrix transform extracts main motion regions of the myocardium as eigen-images by analyzing the statistical information of the images. Second, an RV training model is registered to the eigen-images in order to locate the position of the RV. Third, the training model is adjusted and then serves as an optimized initialization for the segmentation of each image. Finally, based on the initializations, a localized, region-based level set algorithm is applied to segment both epicardial and endocardial boundaries in each echocardiograph. Three evaluation methods were used to validate the performance of the segmentation framework. The Dice coefficient measures the overall agreement between the manual and automatic segmentation. The absolute distance and the Hausdorff distance between the boundaries from manual and automatic segmentation were used to measure the accuracy of the segmentation. Ultrasound images of human subjects were used for validation. For the epicardial and endocardial boundaries, the Dice coefficients were $90.8 \pm 1.7 \%$ and $87.3 \pm 1.9 \%$, the absolute distances were $2.0 \pm 0.42 \mathrm{~mm}$ and $1.79 \pm 0.45 \mathrm{~mm}$, and the Hausdorff distances were $6.86 \pm 1.71$ $\mathrm{mm}$ and $7.02 \pm 1.17 \mathrm{~mm}$, respectively. The automatic segmentation method based on a sparse matrix transform and level set can provide a useful tool for quantitative cardiac imaging.
\end{abstract}

\section{Introduction}

Echocardiography can evaluate the structures and functions of heart ventricles for clinical diagnosis. Image segmentation of the ventricles can provide quantitative measures of heart functions such as ejection fraction (EF). Segmentation of left ventricle (LV) from 2D echocardiography has been widely investigated but the segmentation of right ventricle (RV) is still a research problem (Rudski et al 2010). It has been reported that RV plays an important role in both morbidity and mortality of the patients with signs of cardiopulmonary diseases (Dimitroulas et al 2012). RV segmentation can be challenging because of two main problems: (i) poorer image quality compared to that of LV; and (ii) the irregular geometry of the RV shape, which makes its segmentation difficult in 2D echocardiography. Current efforts for RV segmentation focus on 3D echocardiography (Angelini et al 2001, 2005, Boettger et al 2004). However, increasing evidences from clinical studies emphasize that it

(C) 2013 Institute of Physics and Engineering in Medicine

${ }^{4}$ Author to whom any correspondence should be addressed. bfei@emory.edu, website: http://www.feilab.org. 
is important to evaluate $\mathrm{RV}$ functions through routine $2 \mathrm{D}$ echocardiographic views (Bangalore et al 2007, Rudski et al 2010). RV segmentation can provide diameters, area, myocardium thickness, or fractional area change for routine echocardiographic examinations as well as for other clinical applications such as quantifying the risk stratification and prognosis in stress echocardiography (Bangalore et al 2007). RV segmentation can also be used to calculate the indicator dilution curve of ultrasound contrast agents, which contains the information for the determination of cardiac output, EF, and pulmonary blood volume (Mischi et al 2005).

There are few reports on RV segmentations. On the other hand, there have been numerous efforts devoted to LV echocardiographic segmentation (Noble and Boukerroui 2006). In the previous works, shape prior restriction was emphasized in order to improve the accuracy and reliability of echocardiography segmentations (Dietenbeck et al 2012). Chen et al introduced a shape prior to the geometric active contour algorithm by computing the energy function using both the image gradient and prior shape restrictions. This method was utilized to segment both the epicardial and endocardial boundaries of LV based on the prior shape outlined by experienced echocardiographers (Chen et al 2002, 2007). Taron et al used an ellipse-shaped model to constrain the short axis LV border detection. It was based on the assumption that the short axis endocardium of LV was similar to an ellipse, and which limited its application to only the LV short axis (Taron et al 2004). Recently, Dienbeck et al proposed a geometrically constrained level set algorithm to detect the whole LV myocardium on 2D echocardiography (Dietenbeck et al 2012). They used two hyperquadrics as the shape prior to control the evolving level set contours and an additional thickness term. They proved that this algorithm could be applied to echocardiographic segmentations from any view and also used for the initialization of speckle tracking methods.

Although the shape prior restrictions were useful, these methods required interventional initializations in order to segment each image. It requires much time and effort for physicians to analyze the entire cardiac function in clinical examinations because each echocardiographic series usually contains hundreds of images. Therefore, more efforts have been made to develop the automatic segmentation framework. Bosch et al (2002) improved the active appearance models by the use of an active appearance motion model for automatic border detection in all of the echocardiographic image sequences. They used principal component analysis (PCA) to analyze both the temporal and spatial shape patterns in the sequences. Non-linear intensity normalization was also developed to match the intensity distributions of ultrasound images. In order to analyze the cardiac functions from real-time 3D echocardiography, Zhu et al (2010) segmented both the endocardial and epicardial surfaces of LV using an automatic algorithm that contained a coupled deformable model by considering ultrasound speckle statistics and the myocardium thickness constraints. Meanwhile, Pearlman et al (2012) developed an automatic segmentation method for LV endocardial boundaries based on radio-frequency signals, and which could overcome the inhomogeneities in B-mode images. This method utilized a two-frame linear predictor to consider the spatio-temporal coherence of the data. It was validated by $28,3 \mathrm{D}$ image sequences. Paragios et al (2005) proposed a model-based approach to extract the LV endocardium in each frame of the cardiac cycle. PCA and registration were applied in order to set two, separate models for both systolic and diastolic moments. The segmentation was then realized in two steps: the first step was the rough segmentation to dictate the new model using a linear combination of the systolic and the diastolic models; the second step was to refine the segmentation in order to accurately detect the endocardium boundaries. Zhou et al (2004) used a unified framework for automatic initializations using boosted-shape detection as a generic measurement process. This framework could automatically track the endocardium in ultrasound sequences by propagating the local detection uncertainties of 
multiple shape candidates during shape alignment, fusion with the predicted shape prior, and fusion with subspace constraints. They also presented a machine learning approach called shape regression machine for efficient segmentation of medical images and the method was validated for the $\mathrm{LV}$ endocardium from a B-mode echocardiogram of the apical, 4-chamber view (Zhou 2010). Moreover, based on previous research, a pattern recognition and database-guided segmentation method called AutoEF software (Siemens Medical Solutions, Erlangen, Germany) was developed in the commercial ultrasound scanners. It could automatically detect the endocardium in both end systolic and end diastolic echocardiography and could then track the boundaries in other frames through the cardiac cycle (Rahmouni et al 2008).

Although various methods have been used to segment the LV echocardiography, they cannot be directly applied to RV segmentation because of the poor imaging quality in $2 \mathrm{D}$ echocardiography and because of the irregular shape of the RV. However, RV myocardium segmentations are required for comprehensive RV function analysis. Currently, the RV is primarily segmented manually by experts, which is time-consuming and often requires more than three hours for one image series.

In this study, we propose an automatic segmentation framework for the RV by combining sparse matrix transform (SMT), a training model, and a level set algorithm. The segmentation method can automatically segment both the endocardial and epicardial boundaries of the $\mathrm{RV}$ from a series of $2 \mathrm{D}$ echocardiographic images from coarse recognition to fine segmentations. Our contribution includes (1) the SMT-based level set algorithm, (2) the RV training model, and (3) the application in RV ultrasound segmentation. In this paper, we describe the flowchart of the segmentation framework in section 2, the results and discussions in section 3, and the conclusions in section 4 .

\section{Method}

\subsection{Overview}

Seven healthy subjects were imaged by the SA4-2/24 phased-array transducer and SonixTouch ultrasound system (Ultrasonix Medical Corporation, Richmond, BC, Canada). The imaging parameters are listed as followed: the central frequency of the transducer was 3.5 MHz; the imaging frequency per second was 27; all images were acquired in the standard RV focused apical 4-chamber view (Rudski et al 2010); the dynamic ranges were around 56; the gain was 56\%; the persistence was 0 ; and the imaging data were saved as 8bit log-compress B-mode images. During the imaging periods, the subjects were required to hold their breaths in order to keep a stable probe position. A radiologist specialized in echocardiography (ZC) performed the image acquisitions from the human subjects.

Figure 1 shows the flowchart of the proposed segmentation framework, which is an automatic process from coarse recognition through the entire echocardiographic series to fine segmentation of each image. First, SMT is used to extract eigen-image from the whole echocardiographic series. Second, an RV training model with both epicardial and endocardial boundaries manually defined by a cardiologist (ZC) from 450 images are registered to the eigen-images in order to recognize the $\mathrm{RV}$ regions. This step also determines the corresponding transform relationships between the model and the eigenimage. Third, based on the registration and a tricuspid tracking step, initialization regions are determined for each image in this series by optimizing the shape of the training model. Finally, each myocardium is segmented using a localized, region-based level set algorithm following initializations. 


\subsection{SMT eigen-images and registration}

SMT is currently being successfully applied to face recognition because of its eigendecomposition ability (Cao et al 2011). Compared with other methods, such as PCA, SMT has several advantages: first, it can improve the accuracy of the eigen-decomposition, especially when the number of observations is less than the vector dimension; and second, it runs faster than PCA (Cao et al 2011). Moreover, a graph-based SMT is able to estimate more accurate eigen-images due to the graphical constraint (Bachega et al 2010). Therefore, SMT is introduced to analyze the echocardiographic series containing much less sample data than the image dimension.

Similar to face recognition, one continuous RV echocardiographic series is considered as the data set that contains the similar RV shape in each image. SMT is applied to extract one eigen-image from this data set as the corresponding standardized RV ingredients of the whole series. Figure 2 indicates the SMT process for extracting the eigen-image from the echocardiographic image series. After SMT eigen-decomposition, the components corresponding to the first nine largest eigen-values are chosen as shown in figure 2(b). Each chosen component indicates one connected structure in the echocardiography and corresponds to the moving myocardium tissue in the whole dynamic series. Finally, the eigen-image of the current series (figure 2(c)) is extracted by combining the chosen components. In our case, the number of the first eigen-vectors is selected based on the criteria that the sum of their corresponding eigen-values is bigger than $95 \%$.

SMT extracts the myocardium regions with major motion in the image series and the outcome is the eigen-image. SMT neglects stable structures such as the apex region, as shown in figure 3(b). Because the RV shape differs from other regions such as atriums or $\mathrm{LV}$, it can be easily extracted from the SMT eigen-images by registering it with the mean shape of the training models (figure 3(d)). This registration is based on an automatic similarity registration method (Goshtasby 2005). Transform parameters such as rotation, shift, and scaling, are used in the initialization step to adjust the training model. They restrict the searching region during the initialization because this extracted RV shape indicates the most probable location and shape of the RV in the entire series. Thus, these transform parameters can reduce the calculation time so as to determine the best initialization shape and also keep the model variances in reasonable ranges in order to increase the robustness. Moreover, they can avoid the wrong segmentations in some circumstances such as rotated imaging angles or irregular RV shapes seen in certain diseases (Rudski et al 2010).

Moreover, SMT synthesizes the entire series while considering more information than just directly adapting training models to each image. It can reduce the effects of poor-quality imaging, such as bright noises or missing crucial structures. Moreover, based on the SMT and training models, this approach simulates the expert recognition process where cardiologists follow in their clinical practice to utilize the whole dynamic echocardiographic series rather than just one static image to determine the RV shapes and locations.

\subsection{Optimized initialization and tricuspid tracking}

Similar to the PCA (Cootes et al 1995, Li and Fei 2008, Qu et al 2008, Tsai et al 2003), SMT can be used to capture the main shape components from the training samples. For the training data, both RV endocardial and epicardial boundaries were manually defined from those seen in various echocardiographic series. These boundaries are decomposed by SMT as the mean shape and various components, after which new shapes derived from the training models can be calculated following a linear transform of the largest amount of the SMT components (Paragios et al 2005) 


$$
S=\bar{s}+\sum_{i=1}^{k} b_{i} \times U_{i},
$$

where $S$ is the new shape, $s$ is the mean shape of training model, $U_{i}$ are the eigen-vectors, and $b_{i}$ are the weight parameters related to the eigen-values. $k$ is the number of the selected components, which is set as the sum of selected eigen-values more than $95 \%$.

During the initialization step, the most similar shape to the RV structures in each image is chosen using a genetic algorithm (GA) (Goldberg 1989, Conn et al 1997) through optimizing the weight parameters $b_{i}$ in their searching spaces, which contains initialization, roulette wheel selection, crossover, and mutation steps. The algorithm is shown in figure 4 . GA selects a set of weight parameters to build a new shape which will be transformed following the transform relationship between the mean shape and the SMT eigen-image. Then the energy of the current images in the new shape region will be calculated as the fitness function according to the criteria of the means separation energy function (Yezzi et al 2002):

$$
E_{m s}=-\frac{1}{2}(u-v)^{2}
$$

where $u$ and $v$ are the mean intensities inside and outside of myocardium region in the mean shape (shown as gray region in figure $3(d)$ ), respectively.

Considering the anatomy structures, both the RV and the right atrium are separated by the tricuspid plate. However, the tricuspids are usually unclear and unstable in echocardiography because of their fast motion, and which may lead to incorrect initializations to include the right atrium. Therefore, a speckle tracking algorithm that has proven useful for LV function analysis (Mondillo et al 2011) is introduced into this process to indicate the tricuspid plate position. It begins with the first image at the end of systolic, which can clearly identify the tricuspid plate automatically. The end point of the tricuspid plate at the ventricular septum, which is usually clear during the cardiac period, is identified and is quickly tracked through the entire series by a block matching method with the sum of squared differences criteria (Friemel et al 1995). Based on the tracking results, every initialization is restricted in a reasonable region during energy calculations.

\subsection{Level set segmentation}

After the previous steps, each image has an initialized mask for the following level set segmentation. For example, the gray region in figure 3(e) corresponds to the negative level set, while its epicardial and endocardial boundaries correspond to the zero level set. These masks are not only used as initializations but also as the shape priors. The shape priors are important for the following level set segmentation as their constraints can avoid the level set contours leaking out from weak boundaries, especially when part of the RV structures is missing in some images.

The localized, region-based level set framework, developed by Lankton (Lankton and Tannenbaum 2008), has been applied to the LV myocardium segmentation (Dietenbeck et al 2012). We denote the myocardium boundaries as the zero level set of a signed distance function $\varphi$. Similarly, the level set energy function is designed to contain three aspects: image data, shape prior, and thickness constraint:

$$
E=E_{\text {image }}\left(\phi, I, H_{T}\right)+\lambda E_{\text {shape }}\left(\phi, S, I, H_{T}\right) .
$$


Here, $I$ is the image data, $S$ is the model shape data, and $\lambda$ is a weighted parameter. During the curve evolution and minimization of the energy function $E$, the $E_{\text {image }}$ tends to make $E$ as small as possible when there is evolution nearer to the region edges. Alternatively, the $E_{\text {shape }}$ makes $E$ as large as possible during the evolution farther from its original position. The thickness constraint, $H_{T}$, is defined as a Heaviside function:

$$
H_{T}\left(\phi\left(\bar{x}+\bar{N} \cdot R_{T}\right)\right)= \begin{cases}1, & \phi>0 \\ 0, & \text { otherwise }\end{cases}
$$

and its derivative is defined as:

$$
\delta_{T}\left(\phi\left(\bar{x}+\bar{N} \cdot R_{T}\right)\right)= \begin{cases}1, & \phi=0 \\ 0, & \text { otherwise }\end{cases}
$$

Where $R_{T}$ is the distance between the epicardial and endocardial boundaries at the zero level set point and $N$ is the inward normal of a point $x$. When the distance between both boundaries is smaller than $R_{T}, \varphi\left(x \overline{+} N \cdot R_{T}\right)$ will be positive and $H_{T}\left(\varphi\left(x \overline{+} N \cdot R_{T}\right)\right)$ be 1 . Otherwise it will be 0 . The thickness constraint maintains the minimal thickness between both boundaries as greater than an average value, such as 10 pixels. Based on this definition, it can be drawn that $H_{T} \cdot \delta_{T} \equiv 0$. Therefore, the image data term $E_{\text {image }}$ combining with a thickness constraint can be written as:

$$
E_{\text {image }}=\int_{\Omega_{x}} \delta\left(\phi(\bar{x}) \cdot\left(1-H_{T}^{2}\left(\phi\left(\bar{x}+\bar{N} \cdot R_{T}\right)\right)\right) \int_{\Omega_{y}} B(\bar{x}, \bar{y}) \cdot F(I(\bar{y}), \phi(\bar{y})) \mathrm{d} \bar{y} \mathrm{~d} \bar{x}\right.
$$

Its corresponding energy minimized evolution equation is:

$$
\frac{\partial \phi}{\partial t}(\bar{x})=\delta\left(\phi(\bar{x}) \cdot\left(1-H_{T}^{2}\left(\phi\left(\bar{x}+\bar{N} \cdot R_{T}\right)\right)\right) \cdot \int_{\Omega_{y}} B(\bar{x}, \bar{y}) \cdot \nabla_{\phi} F(I(\bar{y}), \phi(\bar{y})) \mathrm{d} \bar{y}\right.
$$

Similar to the energy calculation of the optimized initialization step, the means separation energy is also applied in level set energy part to calculate the image data energy term.

Therefore, here $F(I(\bar{y}), \phi(\bar{y}))=-\frac{1}{2}\left(u_{x}-v_{x}\right)^{2}$ is the means separation energy function, $u_{x}$ and $v_{x}$ correspond to the inside and outside average intensity values measured in the localized region, and $\delta(\varphi(x))$ is the Dirac function. The function $B(x, \bar{y})$ is a mask to make the localization, defined as

$$
B(\bar{x}, \bar{y})= \begin{cases}1, & \mid \bar{x}-\bar{y} \|<r \\ 0, & \text { otherwise }\end{cases}
$$

where the point $\bar{y}$ is within the localized region with radius $r$ centered at $x$. The definition of the model constraint term $E_{\text {shape }}$ is:

$$
E_{\text {shape }}=\int_{\Omega} \psi^{2} \cdot\|\nabla \phi\| \cdot \delta\left(\phi(\bar{x}) \cdot\left(1+H_{T}^{2}\left(\phi\left(\bar{x}+\bar{N} \cdot R_{T}\right)\right)\right) \mathrm{d} \bar{x} \cdot\right.
$$

Based on the identity $H_{T} \cdot \delta_{T} \equiv 0$, the minimization of (9) can be achieved by evolving $\varphi$, similar to the previous work (Dietenbeck et al 2012): 


$$
\frac{\partial \phi}{\partial t}(\bar{x})=\delta\left(\phi(\bar{x}) \cdot\left(1+H_{T}^{2}\left(\phi\left(\bar{x}+\bar{N} \cdot R_{T}\right)\right)\right) \cdot\left(2 \psi \cdot \nabla_{\phi} \psi \cdot\|\nabla \phi\|+\psi^{2} \cdot \kappa\right)\right.
$$

Where $\psi$ is the shape prior-related function meaning the distance between the zero level set and its corresponding initialization edge of the shape prior. $\kappa$ is the curvature of the evolving zero level set.

\subsection{Segmentation evaluation}

Quantitative assessment of the method is conducted by comparing the segmentation results with the gold standard data obtained from manual segmentation by a cardiologist. The Dice similarity is used as the assessment metrics in myocardium segmentation (Dice 1945). The Dice similarity is computed as follows:

$$
\operatorname{Dice}(S, G)=\frac{2 \operatorname{Area}(S \cap G)}{\operatorname{Area}(S)+\operatorname{Area}(G)}
$$

where $S$ and $G$ represent the pixel set of the segmented regions obtained by the algorithm and the gold standard data, respectively.

Both the mean absolute distance (MAD) (Comaniciu et al 2004) and the Hausdorff distance (HD) (Huttenlocher and Rucklidge 1993) are used as the performance assessment metrics. Suppose A and B are the edges of the automatic and manual results, respectively; and they are represented by point sets: $A=\left\{a_{1}, a_{2}, . . a_{n}\right\}$ and $B=\left\{b_{1}, b_{2}, . . b_{n}\right\}$, MAD and HD are defined as follows:

$$
\begin{gathered}
\operatorname{MAD}(A, B)=\frac{1}{2}\left\{\frac{1}{m} \sum_{i=1}^{m} d\left(a_{i}, B\right)+\frac{1}{n} \sum_{j=1}^{n} d\left(b_{i}, A\right)\right\} \\
\mathrm{HD}(A, B)=\max \left\{\max _{i}\left\{d\left(a_{i}, B\right)\right\}, \max _{j}\left\{d\left(b_{j}, A\right)\right\}\right\}
\end{gathered}
$$

where $d\left(a_{i}, B\right)=\min _{j}\left\|b_{j}-a_{i}\right\|$, MAD is a global measurement, and $\mathrm{HD}$ is a similarity for their locals.

\subsection{Implementation}

Some implementation details are described below. The algorithm was implemented in MATLAB language. The reproduction of SMT covariance estimation was based on the previous work (Bachega et al 2010, Cao et al 2011, Qin et al 2013). On the initialization step, the criterion for choosing the training model parameter $k$ was that the sum of the first $k$ eigen-values was bigger than $95 \%$, which was 4 for our training model. Its corresponding weight parameters $b_{i}$ were set in the range $[-0.5,0.5]$ for the GA initialization and searching. The GA crossover and mutation parameters were 0.8 and 0.1 , respectively. Its stop condition was that the fitness function kept stable for more than 20 generations or when the maximum generation reaches. On the bock matching step, the block size is $50 \times 50$ pixels and the maximum searching range is 10 pixels. Because this step only tracked one block, it only costs $30 \mathrm{~s}$ for 120 continuous images. The weighted parameter $\lambda$ in the level set, which is related to shape prior term, was set as 0.4 , because RV segmentation in 2D echocardiography needed more shape prior to avoid the poorer imaging quality. 


\section{Results}

Eight dynamic image series containing 1,158 images were used as the testing data, which were different from those images applied in the training data. RVs in these series were segmented by our proposed method throughout the entire cardiac cycles. The automatic segmentation results were evaluated by the manual gold standards using the DICE overlap ratios, MAD, and $\mathrm{HD}$ scores.

\subsection{Myocardium segmentation results}

Figure 5 presents a segmented echocardiography during one cardiac cycle. Both green, solid lines correspond to the epicardial and endocardial boundaries of the RV myocardium, respectively; and their shapes change along the different heart-beating phases. Additionally, the red dots in these images are the tricuspids indicating the points tracked by the speckle tracking method. As shown in the image, the lateral walls of the RV are vague, and which is a normal phenomenon in RV echocardiographic images affected by the lung or other structures, although this automatic segmentation method still provides reasonable segmentations. However, these poor RV imaging qualities, especially in the cases where the lateral wall entirely disappears, increase the disagreement between both automatic segmentation and the manual segmentations. The segmentation results of these selected echocardiographic images were evaluated using the Dice, MAD, and HD methods. These evaluations results for both the epicardial and endocardial segmentations are shown in table 1 , where the mean epicardial Dice is $90.8 \pm 1.7 \%$, the mean endocardial Dice is $87.3 \pm$ $1.9 \%$, the mean epicardial MAD is $2.0 \pm 0.42 \%$, the mean endocardial MAD is $1.79 \pm$ $0.45 \%$, the mean epicardial HD is $6.86 \pm 1.71 \mathrm{~mm}$, and the mean endocardial HD is $7.02 \pm$ $1.17 \mathrm{~mm}$.

\subsection{Comparison of three segmentation frameworks}

Currently, there were few automatic approaches that were applied in RVsegmentation in 2D echocardiography series. The main approach was still manual segmentation by eachocardiologists. On the other hand, level set has been applied in the LV segmentations and 3D RV segmentations in echocardiography (Angelini et al 2005, Noble and Boukerroui 2006). Therefore, we utilized the basic region-based level set with arbitrary initialization as method 1 and its improvement with optimized initializations as method 2 to validate the performance of our proposed method. We compared the abilities of the three different segmentation frameworks in one image series. The details of these three methods are (I) the classic, localized, region-based level set with means separation energy, (II) the same level set but with an optimized initialization, and (III) our proposed method with an optimized initialization and a shape prior for the segmentation of RV from echocardiographic images. After the same iterations, the results are shown in figure 6, which indicate that specific initialization improves the level set segmentation because the localized, regions-based level set method can be sensitive to initialization (Lankton and Tannenbaum 2008). Without any shape prior constraint, the first two segmentation methods did not perform well (figures 6(b2) and (c2)) because the RV is vague and has complicated structures in echocardiographic images. However, our proposed method performs well and the automatic segmentation result is close to the manual result (figure 6(d2)). Moreover, comparing with the gold standards, their mean evaluations values are: 42.3\% (DICE), $25.48 \mathrm{~mm}$ (MAD), and $83.94 \mathrm{~mm}$ (HD) for method I; 72.8\% (Dice), $4.08 \mathrm{~mm}$ (MAD), $22.02 \mathrm{~mm}$ (HD) for method II; and $87.5 \%$ (Dice), $2.20 \mathrm{~mm}$ (MAD), and $7.52 \mathrm{~mm}$ (HD) for method III. These results indicate that the procedures of specific initializations and shape priors are necessary for the automatic RV segmentations in echocardiographic series. 


\subsection{The effect of SMT-based transform on RV segmentation}

Figure 7 compares the results from GA-optimized initialization with and without SMTbased transform. Figure 7(d) shows the result of GA-optimized segmentation that directly searches in the current image without any SMT transform relationship constraint. Alternatively, figure 7(f) shows the result of the segmentation based on the initialization with SMT-based transform. The SMT-based transform increases the accuracy of RV segmentations by considering the information of the entire series rather than only that of the current image. This is because the locations, rotations, and scales of RV regions usually vary during different imaging angles or with different study subjects (Rudski et al 2010). Without this transform, the initialization process can detect the wrong tissue such as the right atrium (figure 7(d)). This problem also occurs when using the LV automatic segmentation methods which only consider the current image information (Rahmouni et al 2008). Moreover, the GA-optimized initialization process takes much less time after restricting the transform relationships, because it only needs to optimize the weight parameters in smaller searching ranges.

\subsection{Robustness evaluation}

We evaluated the robustness of the segmentation method. Five different images were selected and were segmented 10 times following the same parameters. Dice, MAD, and HD were used to evaluate the segmentation by comparing the manual and automatic segmentation results. As shown in figure 8, the standard deviations of the Dice scores for both endocardial and epicardial boundaries are approximately $0.5 \%$. Moreover, the standard deviations of MAD are less than $0.2 \mathrm{~mm}$ and those of HD are less than $0.8 \mathrm{~mm}$. These results indicate the strong robustness of the proposed segmentation method. The most repeatable differences are derived from the GA-optimized initialization step because the GA searching process was randomly selected. However, this robustness can be improved by the use of more searching steps, by a more constrained searching range, and by combining SMT-based transform.

\section{Discussion}

Automatic segmentation of the RV on ultrasound images is a challenging task. Although various methods have been proposed for the segmentation of the LV, they cannot be directly applied to segment the RV. The proposed segmentation method is able to segment both the endocardial and epicardial boundaries at the same time. One observation which should be noticed is that the general endocardial Dice scores are lower than the epicardial scores. One explanation for which is that the endocardial region is smaller and more irregular than the epicardial region, especially in the apical regions. Moreover, the papillary tissue also affects the endocardial segmentation accuracy for both the manual and automatic results.

The proposed RV myocardium segmentation method focuses on the routine 2D imaging examinations in a standard RV focused apical 4-chamber view. After the endocardium and epicardium are segmented, other parameters, such as RV diameters, area, and myocardium thickness, can be derived from the segmented images. The changes in the fractional area can also be derived for routine echocardiographic examinations. These quantitative parameters can be further explored for risk stratification and prognosis in stress echocardiography. The segmentation method can also be used to calculate the indicator dilution curve of ultrasound contrast agents, which is widely used in the intensive care unit and operating room for cardiac parameter measurements. Although this method is proposed for 2D RV segmentation, it can be extended to 3D RV echocardiographic volumes because all steps including SMT, optimized initialization, and region-based level set in the proposed framework can be applied to 3D data. Based on segmented 3D data, accurate RV functional 
parameters such as end dilation volume, end systolic volume and EF can be calculated for clinical applications. When the segmentation method is extended to 3D RV images, the establishment of the training model may need efforts from experts for manual segmentation. Another challenge could be the fact that the 3D structure of RV can be complicated and that the image resolution in 3D can be low. Although it may take some efforts to extend the proposed automatic RV segmentation method to 3D echocardiography series, the framework can be adapted and implemented for 3D imaging applications.

During the robustness evaluation, we randomly selected five images from different series and each image was segmented 10 times and the Dice, MAD and HD parameters were calculated. The results indicated the robustness for 10-time segmentations on one same image. We considered that five random selected images were enough for the robustness evaluation because the purpose of this step is to test whether the segmentation was robust for the same image.

The implementation of the algorithm can be improved in the future. Currently, the total time for one series of 150 continuous images is $3-4 \mathrm{~h}$. There were several reasons leading to this computation time: (1) the SMT needed computing time that depends on the image size; (2) the images were initialized and segmented one after another; (3) the current algorithm was implemented in MATLAB that was not efficient enough; and (4) we used a desktop computer workstation for this implementation. The level set segmentation takes less than $2 \mathrm{~s}$ for each image. In order to solve this problem, the computation time can be significantly reduced if the algorithm is implemented in $\mathrm{C} / \mathrm{C}++$ as compared to the current MATLAB codes. Furthermore, after SMT processing, parallel computing can be used to segment multiple images at the same time using multiple CPU processors, which will dramatically increase the computing speed. This paper focused on the feasibility and accuracy of the algorithmic procedure. We plan the parallel processing and $\mathrm{C}++$ implementation in our future work.

\section{Conclusions}

We developed and evaluated an automatic segmentation framework for the right ventricle on echocardiographic images. This framework can automatically segment both epicardial and endocardial boundaries obtained from a continuous echocardiography series. The segmentation method combines sparse matrix transform, a training model, and a level set algorithm. Sparse matrix transform is used to extract the eigen-images of the echocardiographic series in order to automatically identify the myocardium region in the current series. Based on the extracted eigen-images, an RV training model is registered and optimized as an initialization for the following localized, region-based level set segmentation. Experimental results from human subject data demonstrated the performance of the proposed segmentation framework for the right ventricle on ultrasound images. It can also be applied to other imaging modalities such as cardiac MR imaging. The segmentation method could also have potential applications for left ventricle segmentation as well as other organs.

\section{Acknowledgments}

This research is supported in part by NIH grants R01CA156775 and R21CA176684, Georgia Research Alliance Distinguished Scientist Award, and the Emory Molecular and Translational Imaging Center (NIH P50CA128301). 


\section{References}

Angelini ED, Homma S, Pearson G, Holmes JW, Laine AF. Segmentation of real-time threedimensional ultrasound for quantification of ventricular function: a clinical study on right and left ventricles. Ultrasound Med Biol. 2005; 31:1143-58. [PubMed: 16176781]

Angelini ED, Laine AF, Donis J, Gersony D, Homma S. Quantification of right and left ventricular function with real-time three-dimensional ultrasound. Proc 23rd Annu Int Conf IEEE Engineering in Medicine and Biology Society. 2001; 3:2587-90.

Bachega LR, Cao GZ, Bouman CA. Fast signal analysis and decomposition on graphs using the sparse matrix transform. IEEE Int Conf on Acoustics, Speech, and Signal Processing. 2010:5426-9.

Bangalore S, Yao SS, Chaudhry FA. Role of right ventricular wall motion abnormalities in risk stratification and prognosis of patients referred for stress echocardiography. J Am Coll Cardiol. 2007; 50:1981-9. [PubMed: 17996564]

Boettger T, Wolf I, Kunert T, Mottl-Link S, Hastenteufel M, De Simone R, Meinzer HP. Semiautomatic 3D-segmentation of live-3D echocardiographic images. Comput Cardiol (2004). 2004; 31:73-6.

Bosch JG, Mitchell SC, Lelieveldt BPF, Nijland F, Kamp O, Sonka M, Reiber JHC. Automatic segmentation of echocardiographic sequences by active appearance motion models. IEEE Trans Med Imaging. 2002; 21:1374-83. [PubMed: 12575874]

Cao GZ, Bachega LR, Bouman CA. The sparse matrix transform for covariance estimation and analysis of high dimensional signals. IEEE Trans Image Process. 2011; 20:625-40. [PubMed: 20813641]

Chen Y, Huang F, Tagare HD, Rao M. A coupled minimization problem for medical image segmentation with priors. Int J Comput Vis. 2007; 71:259-72.

Chen YM, Tagare HD, Thiruvenkadam S, Huang F, Wilson D, Gopinath KS, Briggs RW, Geiser EA. Using prior shapes in geometric active contours in a variational framework. Int J Comput Vis. 2002; 50:315-28.

Comaniciu D, Zhou XS, Krishnan S. Robust real-time myocardial border tracking for echocardiography: an information fusion approach. IEEE Trans Med Imaging. 2004; 23:849-60. [PubMed: 15250637]

Conn AR, Gould N, Toint PL. A globally convergent Lagrangian barrier algorithm for optimization with general inequality constraints and simple bounds. Math Comput. 1997; 66:261-88.

Cootes TF, Taylor CJ, Cooper DH, Graham J. Active shape models-their training and application. Comput Vis Image Underst. 1995; 61:38-59.

Dice LR. Measures of the amount of ecologic association between species. Ecology. 1945; 26:297302.

Dietenbeck T, Alessandrini M, Barbosa D, D’hooge J, Friboulet D, Bernard O. Detection of the whole myocardium in 2D-echocardiography for multiple orientations using a geometrically constrained level-set. Med Image Anal. 2012; 16:386-401. [PubMed: 22119489]

Dimitroulas T, Mavrogeni S, Kitas GD. Imaging modalities for the diagnosis of pulmonary hypertension in systemic sclerosis. Nature Rev Rheumatol. 2012; 8:203-13. [PubMed: 22310183]

Friemel BH, Bohs LN, Trahey GE. Relative performance of two-dimensional speckle-tracking techniques: normalized correlation, non-normalized correlation and sum-absolute-difference. IEEE Ultrasonics Symp Proc. 1995; 2:1481-4.

Goldberg, DE. Optimization and Machine Learning. Reading, MA: Addison-Wesley; 1989. Genetic algorithms in search.

Goshtasby, A. Remote Sensing, and Industrial Applications. Hoboken, NJ: Wiley; 2005. 2D and 3D image registration for medical.

Huttenlocher DP, Rucklidge WJ. A multiresolution technique for comparing images using the hausdorff distance. IEEE Computer Society Conf on Computer Vision and Pattern Recognition: Proc. 1993:705-6.

Lankton S, Tannenbaum A. Localizing region-based active contours. IEEE Trans Image Process. 2008; 17:2029-39. [PubMed: 18854247] 
Li K, Fei BW. A deformable model-based minimal path segmentation method for kidney MR images. Proc SPIE. 2008; 6914:F9144-F.

Mischi M, Kalker AA, Korsten HH. Cardiac image segmentation for contrast agent videodensitometry. IEEE Trans Biomed Eng. 2005; 52:277-86. [PubMed: 15709665]

Mondillo S, et al. Speckle-tracking echocardiography: a new technique for assessing myocardial function. J Ultrasound Med. 2011; 30:71-83. [PubMed: 21193707]

Noble JA, Boukerroui D. Ultrasound image segmentation: a survey. IEEE Trans Med Imaging. 2006; 25:987-1010. [PubMed: 16894993]

Paragios N, Jolly MP, Taron M, Ramaraj R. Active shape models and segmentation of the left ventricle in echocardiography. Proc Scale Space and Pde Methods in Computer Vision. 2005; 3459:131-42.

Pearlman PC, Tagare HD, Lin BA, Sinusas AJ, Duncan JS. Segmentation of 3D radio frequency echocardiography using a spatio-temporal predictor. Med Image Anal. 2012; 16:351-60. [PubMed: 22078842]

Qin X, Cong Z, Halig LV, Fei B. Automatic segmentation for right ventricle on ultrasound images using sparse matrix transform and level set. Proc SPIE. 2013; 8669:86690Q.

Qu W, Huang XL, Jia YY. Segmentation in noisy medical images using PICA model based particle filtering. Medical Imaging 2008: Image Processing, Pts 1-3 Proc SPIE. 2008; 6914:I9143I.

Rahmouni HW, Ky B, Ptappert T, Duffy K, Wiegers SE, Ferrari VA, Keane MG, Kirkpatrick JN, Silvestry FE, Sutton MSJ. Clinical utility of automated assessment of left ventricular ejection fraction using artificial intelligence-assisted border detection. Am Heart J. 2008; 155:562-70. [PubMed: 18294497]

Rudski LG, Lai WW, Afilalo J, Hua LQ, Handschumacher MD, Chandrasekaran K, Solomon SD, Louie EK, Schiller NB. Guidelines for the echocardiographic assessment of the right heart in adults: a report from the American society of echocardiography endorsed by the european association of echocardiography, a registered branch of the European society of cardiology, and the Canadian society of echocardiography. J Am Soc Echocardiogr. 2010; 23:685-713. [PubMed: 20620859]

Taron M, Paragios N, Jolly MP. Border detection on short axis echocardiographic views using a region based ellipse-driven framework. Proc Medical Image Computing and Computer-Assisted Intervention-Miccai. 2004; 3216(1):443-50.

Tsai A, Yezzi A, Wells W, Tempany C, Tucker D, Fan A, Grimson WE, Willsky A. A shape-based approach to the segmentation of medical imagery using level sets. IEEE Trans Med Imaging. 2003; 22:137-54. [PubMed: 12715991]

Yezzi A, Tsai A, Willsky A. A fully global approach to image segmentation via coupled curve evolution equations. J Vis Commun Image Represent. 2002; 13:195-216.

Zhou SK. Shape regression machine and efficient segmentation of left ventricle endocardium from 2D B-mode echocardiogram. Med Image Anal. 2010; 14:563-81. [PubMed: 20494610]

Zhou XS, Comaniciu D, Xie B, Cruceanu R, Gupta A. A unified framework for uncertainty propagation in automatic shape tracking. Proc IEEE Computer Society Conf on Computer Vision and Pattern Recognition. 2004; 1:872-9.

Zhu Y, Papademetris X, Sinusas AJ, Duncan JS. A coupled deformable model for tracking myocardial borders from real-time echocardiography using an incompressibility constraint. Med Image Anal. 2010; 14:429-48. [PubMed: 20350833] 


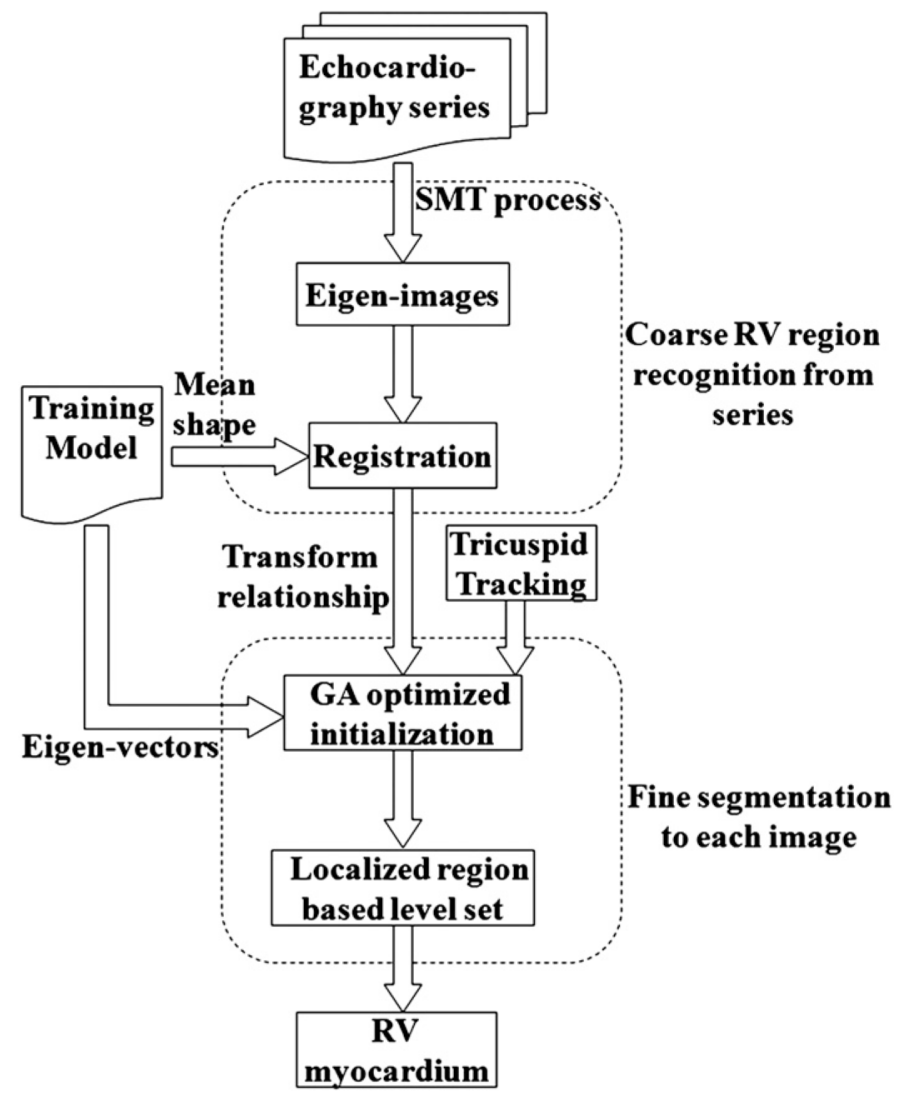

Figure 1.

Flowchart of the automatic echocardiography segmentation framework from general recognition to fine segmentation. 


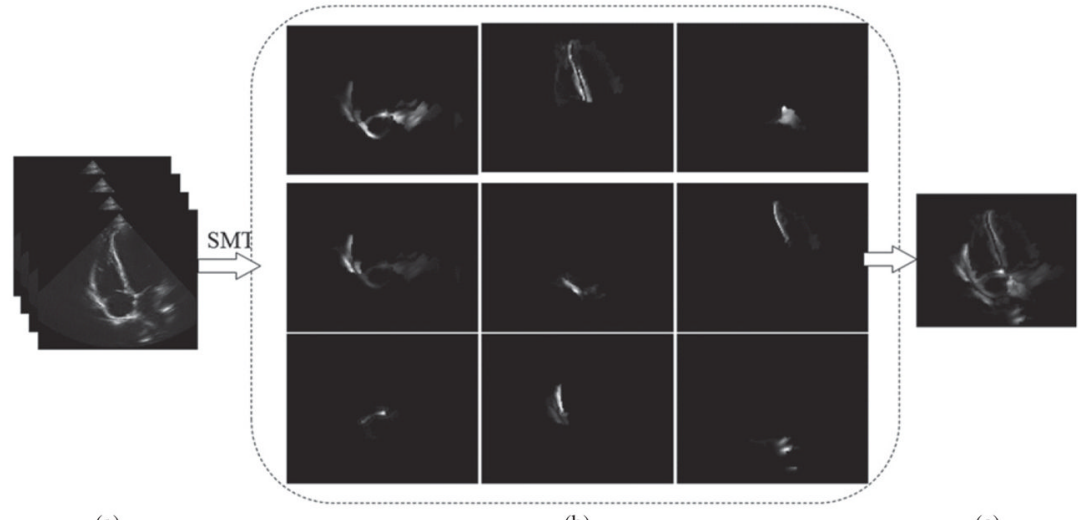

(a)

(b)

(c)

Figure 2.

The eigen-image extracted from echocardiographic series. (a) Echocardiographic series. (b) The first nine components of SMT eigen-decomposition, which captured the local spatial structure of the myocardium. (c) The eigen-image of this series by summing up all absolute values of those components in (b), where the intensity corresponds to the sum value. 


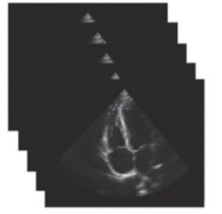

(a)

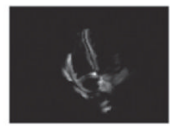

(b)

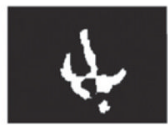

(c)

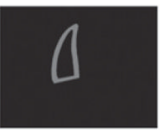

(d)

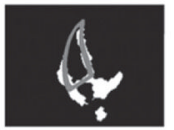

(e)

Figure 3.

The RV recognition from one echocardiographic series. (a) Echocardiographic series. (b) The eigen-image extracted from the image series by SMT. (b) Corresponding black-white image of eigen-image where the white region indicates the most probable motion region of the myocardium. (d) The mean shape of the training model. (e) The registration result of the training model and the SMT eigen-image, whose transform relationships contain shifts, rotation, and scaling. 


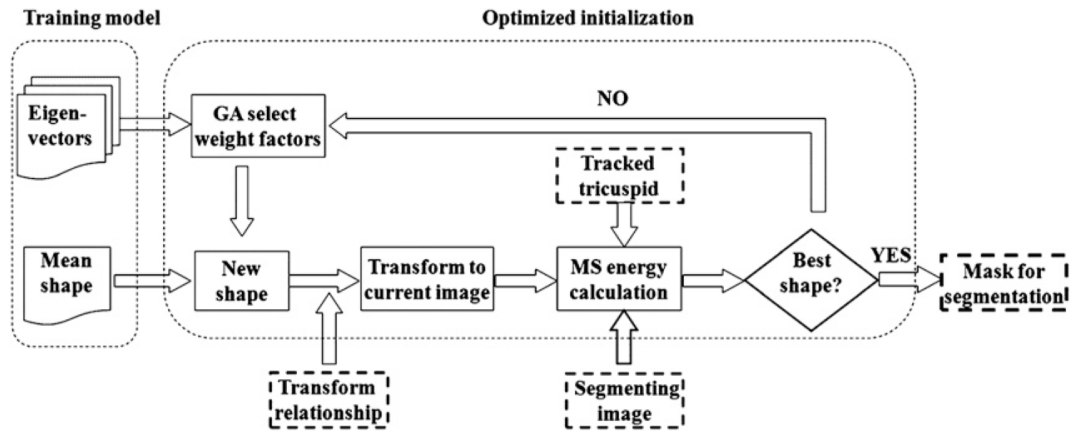

Figure 4.

Illustrations of the GA optimized initialization to select the best shape from the training model. 

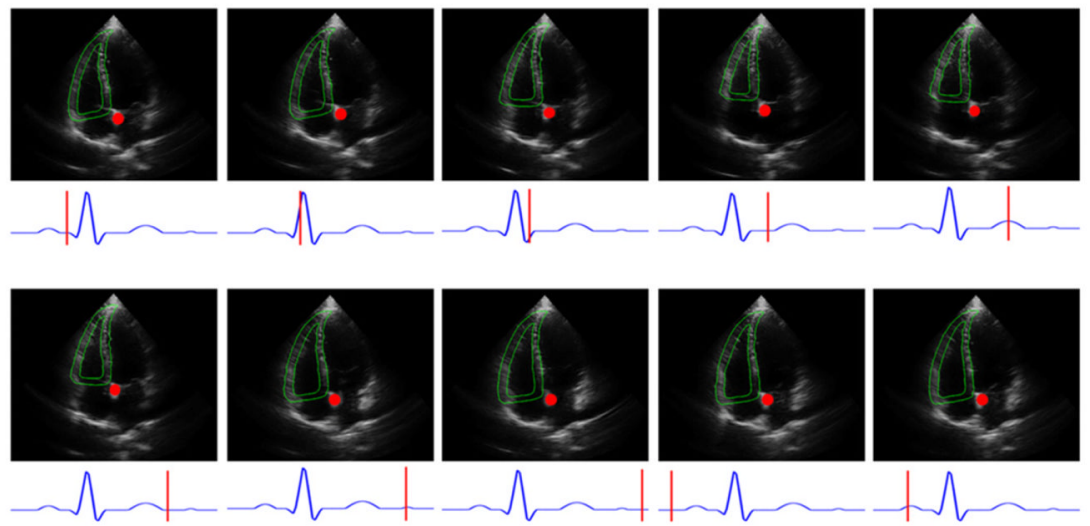

Figure 5.

The segmented echocardiographic images in one beating period, where the green solid lines are both epicardium and endocardium. The red dots in these images are the tricuspid tracking points. The ECG curves below images indicate their corresponding phases in cardiac cycles. The general dynamic ranges utilized in our experiment were around 56 and they were log-compressed B-mode images. 

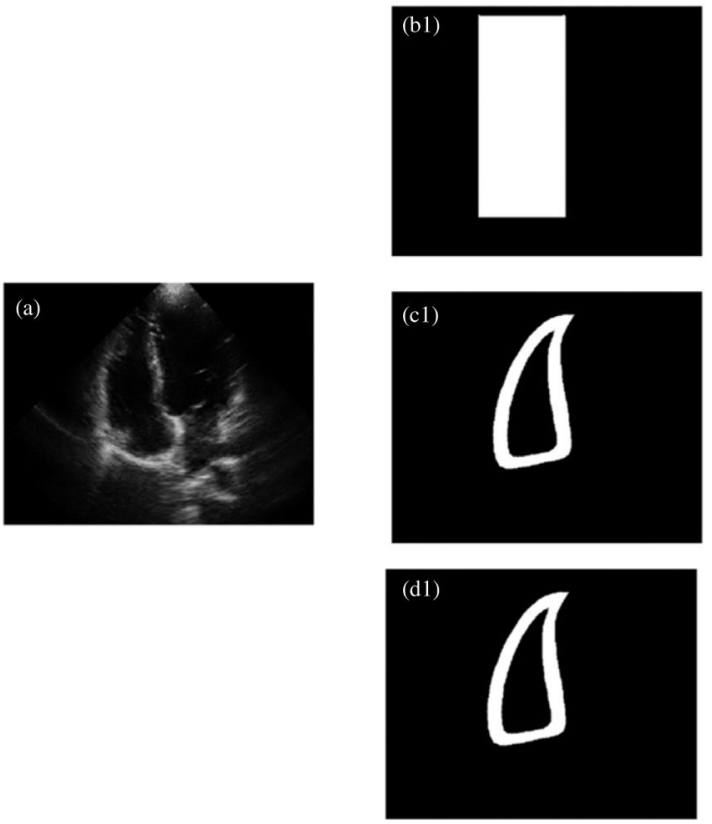
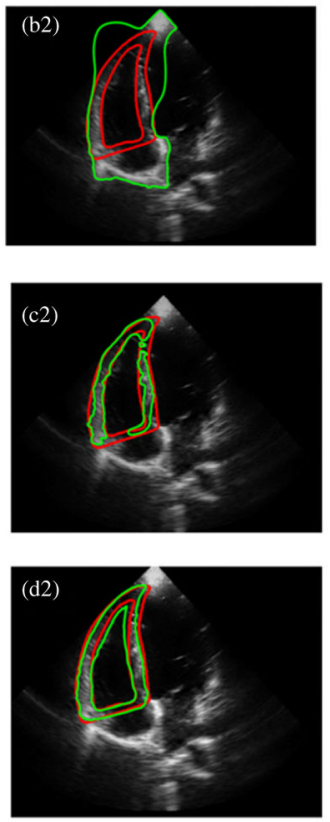

Figure 6.

The results are segmented by different frameworks where the green solid lines are the detected boundaries of the myocardium and the red solid lines are their corresponding gold standard. (a) is an ultrasound image needing segmentation. (b2) is the result segmented by the localized, region-based level set with an arbitrary initialization mask (b1). (c2) is the result segmented by the localized, region-based level set with an optimized initialization mask (c1), but without any restriction. (d2) is the result segmented by the localized, regionbased level set with an optimized initialization mask (d1) and also restricted by the mask. 

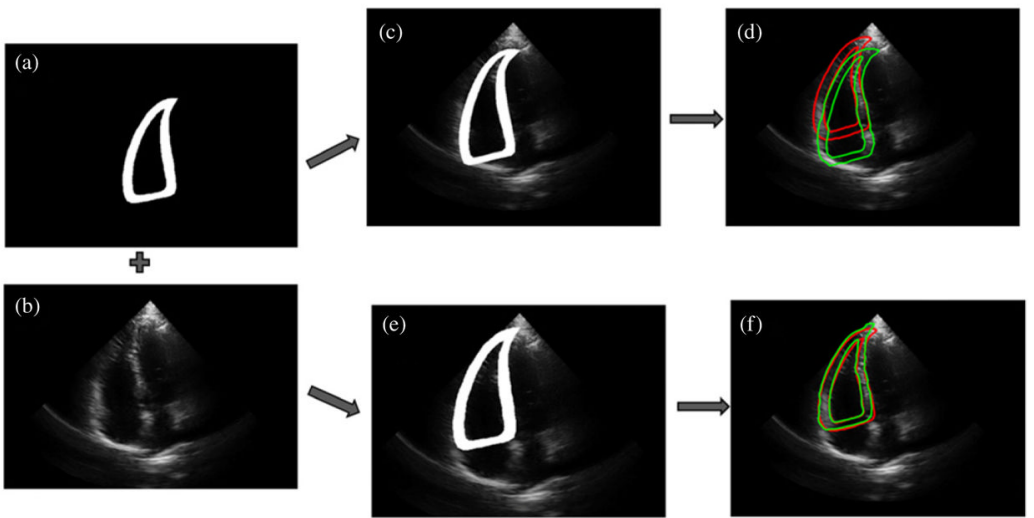

Figure 7.

GA optimized initialization and segmentation results without and with SMT-based transform, where green lines are segmented results and red lines are the corresponding gold lines. (a) Mean shape of the training model. (b) Echocardiographic image to be segmented, where locations, rotations and scales of RV regions are usually changeable because of different imaging angles and different subjects. (c) GA optimized mask without SMT-based transform. (d) Segmented result based on mask (c). (e) GA optimized mask with SMT-based transform. (f) Segmented result based on mask (e). 

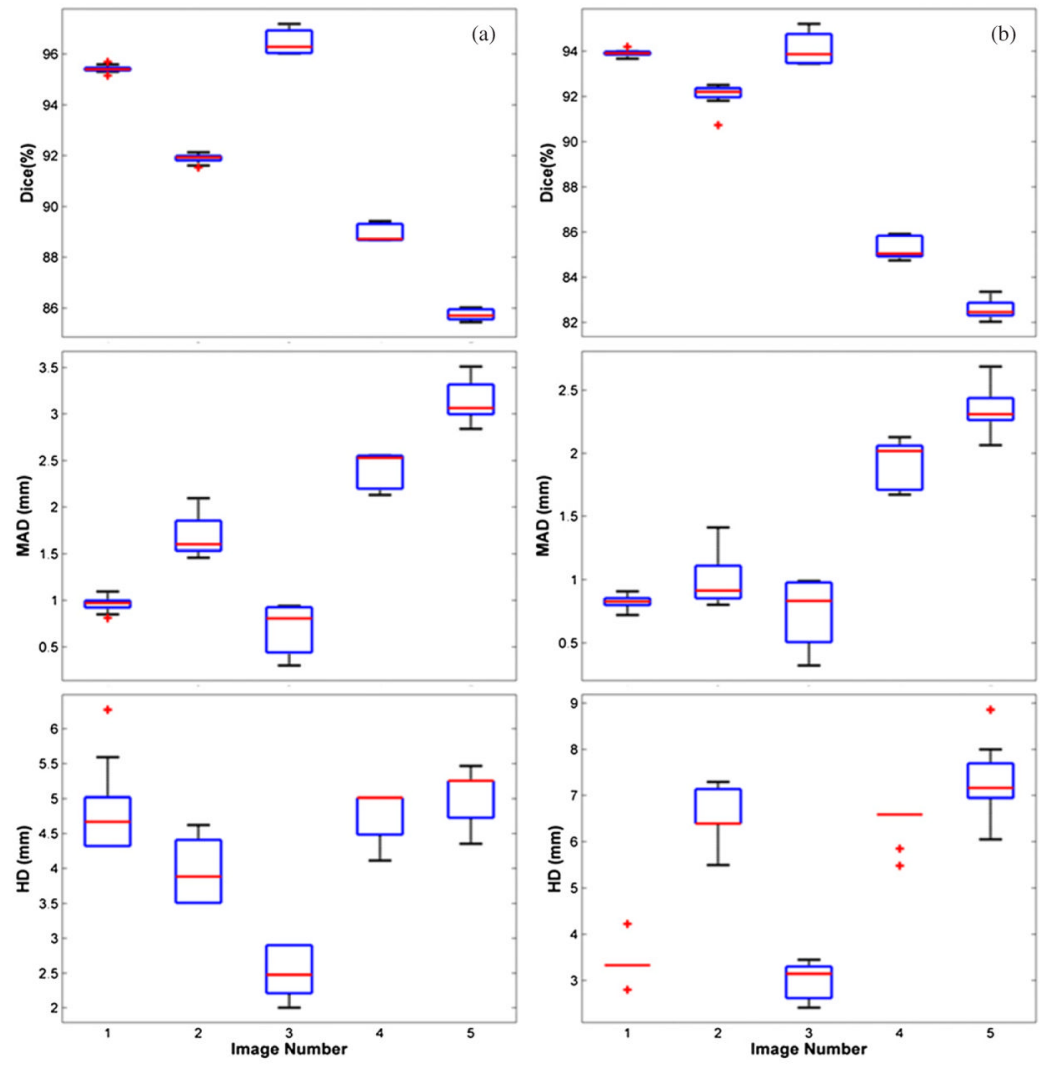

Figure 8.

Robustness of the segmentation method is validated by five different images, each of which was segmented 10 times. Their results are evaluated by manual results using Dice, MAD and HD, respectively. (a) Results of epicardium. (b) Results of endocardium. On each plotted box, the central mark is the median, the edges of the box are the 25th and 75th percentiles, the whiskers extend to the most extreme data points not considered outliers, and outliers are plotted individually. 
